\title{
Welcome Announcement
}

The editorial board of "Discrete Event Dynamic Systems: Theory and Applications" welcomes the following new members to join the board as Associate Editors:

Professor Bart De Schutter, Delft University of Technology, Netherlands Professor Qing-Shan Jia, Tsinghua University, China

Professor Jianfeng Mao, Nanyang Technological University, Singapore

Professor Thomas Moor, Friedrich-Alexander Universität Erlangen-Nürnberg, Germany

Xi-Ren Cao, Editor-in-Chief

November 26, 2012 